WIENER SLAVISTISCHES JAHRBUCH, Band 55/2009, 13-24

(C) 2009 by Österreichische Akademie der Wissenschaften, Wien

PETER B ACHMAIER

\title{
Die Kulturpropaganda der Volksrepublik Bulgarien in Österreich
}

\section{Die ANNÄHERUNG ZWISChen ÖSTERREICH UND BULGARIEN}

Österreich war seit der Unterzeichnung des Staatsvertrags von 1955 ein neutraler Staat, der keinem militärischen Bündnis angehörte, aber ein westlich-demokratisches Gesellschaftsmodell verwirklichte. Die österreichische Regierung entfaltete unter Bruno Kreisky als Außenminister (1959-1965) und als Bundeskanzler (19701983) eine aktive Ostpolitik, in der Österreich als Vermittler und Brücke zwischen Ost und West fungieren sollte. Kreisky konzentrierte sich vor allem auf die Verbesserung der Beziehungen zu den östlichen Nachbarländern. Kreisky führte mit diesen Ländern bilaterale Verhandlungen, um in Mitteleuropa die Entspannung zu fördern, und konnte dadurch die internationale Rolle Österreichs stärken. ${ }^{1}$

Kreisky hielt an der österreichischen Neutralität fest, vor allem aus Rücksicht auf die Sicherheitsinteressen der Sowjetunion, während die Österreichische Volkspartei eine Integration in die Europäische Wirtschaftsgemeinschaft, die spätere Europäische Union, anstrebte. Kreisky nützte die Periode der Entspannung zwischen den beiden Supermächten aus, insbesondere nach dem Helsinkiabkommen von 1975, um eine relativ souveräne Außenpolitik zu betreiben und das internationale Ansehen Österreichs zu erhöhen. Der österreichische Beitrag zur Entspannung zeigt, welche Möglichkeiten auch kleine Staaten in der internationalen Politik gegenüber den Großmächten haben. ${ }^{2}$

Wien wurde von der bulgarischen Führung als Zentrum ihrer Kulturpolitik im Westen ausgewählt, weil es in der Vergangenheit fast immer enge positive politische

${ }^{1}$ Otmar Höll, The Foreign Policy of the Kreisky Era, Contemporary Austrian Studies 2, 1993, 39-42, und Oliver Rathkolb, Bruno Kreisky: Perspektives of Top Level U.S. Foreign Policy Decision Makers, 1959-1983, Contemporary Austrian Studies 2, 1993, 132134.

2 Rathkolb, Bruno Kreisky, 134. 
und kulturelle Beziehungen zwischen beiden Ländern gab, weil ein Teil der bulgarischen kulturellen und wissenschaftlichen Elite, die damals die führenden Positionen an der Sofioter Universität oder an der Akademie der Wissenschaften innehatten, noch an österreichischen Universitäten studiert hatte, und weil es ein neutrales Land war, das nicht dem westlichen Bündnissystem angehörte.

Die Initiative ging von Bulgarien aus, d. h. nicht von Wien und auch nicht von Moskau. Die bulgarische Kulturoffensive war sicherlich nur mit Zustimmung Moskaus möglich, denn die gesamte internationale Politik des Ostblocks wurde von der sowjetischen Führung koordiniert; aber es wäre zu einfach, Bulgarien nur als Werkzeug der sowjetischen Strategie zu sehen. Bulgarien konnte gerade auf kulturellem Gebiet, das nicht im selben Ausmaß politisch kontrolliert wurde, mit Zustimmung der Sowjetunion eine eigene Initiative entfalten.

Die Errichtung des bulgarischen Kulturzentrums in Wien war ferner nur möglich, weil die führenden österreichischen politischen, kulturellen und wissenschaftlichen Kreise für die bulgarische Initiative offen waren. Die Politik der österreichischbulgarischen Zusammenarbeit wurde von beiden großen österreichischen Parteien, ÖVP und SPÖ, getragen. Die Politik der Entspannung wurde bereits in den 60er Jahren von Politikern beider Parteien begonnen. In den 70er und 80er Jahren war vor allem die sozialistische Staatsführung Träger dieser Zusammenarbeit, aber die ÖVP hatte mit dem Staatsbesuch von Bundeskanzler Klaus 1957 dieser Entwicklung einen wesentlichen Impuls gegeben und war auch später durch ihre führenden Funktionäre immer in diese Zusammenarbeit eingebunden. Aber auch die FPÖ unterstützte in der Zeit ihrer ersten Regierungsbeteiligung 1983-1986 diese Politik. ${ }^{3}$

Die Annäherung zwischen Österreich und Bulgarien erreichte rasch einen Punkt, der eine Befassung der höchsten Staatsorgane erforderlich machte. Im Anschluss an den erfolgreichen Besuch des Bundeskanzlers Dr. Josef Klaus in Bulgarien vom 17. bis 21. Oktober 1967, bei dem eine Verstärkung des Kulturaustausches vereinbart wurde, legte das bulgarische Außenministerium am 10. November 1967 dem Zentralkomitees der BKP einen Plan für die Zusammenarbeit mit Österreich vor ${ }^{4}$.

Auf Vorschlag des Außenministers Ivan Bašev und des Leiters der Abteilung „Außenpolitik und internationale Beziehungen“ des ZK der BKP Konstantin Telalov beschloss das Politbüro des ZK der BKP am 16. Jänner 1968 das Programm einer bedeutenden Erweiterung und Vertiefung der wirtschaftlichen, handelsmäßigen und wissenschaftlich-technischen Zusammenarbeit mit Österreich. Das Dokument sah

${ }^{3}$ Bericht des Botschaftsrates Veselin Antov von der bulgarischen Botschaft in Wien über den Besuch von Bundeskanzler Klaus in Bulgarien, Oktober 1967, CDA, F 378-B, a. e. $808,1.61$.

4 Vorschlag von Außenminister Ivan Bašev vom 10. 11. 1967 an das ZK der BKP, CDA, Fonds 1, op. 35, a. e. 12. 
u. a. einen systematischen Kulturaustausch vor. ${ }^{5}$ Dieses Programm wurde durch einen weiteren Beschluss des Politbüros vom 27. Mai 1969 „Über die grundlegenden Richtungen und Hauptaufgaben der Außenpolitik der VR Bulgarien nach Regionen und Ländern“ ergänzt. ${ }^{6}$

Die folgende „Erweiterung und Vertiefung der Zusammenarbeit“ zwischen Österreich und Bulgarien, die in den siebziger und achtziger Jahren ein für zwei Länder mit unterschiedlichen politischen Systemen ungewöhnlich hohes Niveau erreichte, beruhte auf diesen beiden Beschlüssen des Politbüros vom Jänner 1968 und Mai 1969. Am 22. 12. 1970 fand im Außenministerium in Sofia eine bedeutende vertrauliche Besprechung des Kollegiums des Außenministeriums in Anwesenheit des Ministers Ivan Bašev statt, auf der die Ergebnisse der bisherigen Politik diskutiert und Richtlinien für die weitere Entwicklung der Beziehungen zu Österreich ausgearbeitet wurden ${ }^{7}$.

Das Kollegium des Außenministeriums kritisierte, dass die Beschlüsse des Politbüros vom Jänner 1968 und Mai 1969 hinsichtlich der Beziehungen zur österreichischen Regierung, der Vermittlung politischer Maßnahmen und des Sammelns von Informationen unzureichend erfüllt wurden. Die bulgarische Botschaft in Wien erhielt den Auftrag, den Einfluss Bulgariens in Österreich zu verstärken, insbesondere durch den Aufbau von Beziehungen zu führenden Politikern und Vertretern des öffentlichen Lebens (unter diesen wurde Kardinal Dr. Franz König als einziger persönlich genannt $)^{8}$.

\section{Die Kulturpolitik LuUdmila Ž̆}

Mit der Entwicklung des Helsinkiprozesses wuchs auch die Rolle und die Bedeutung des Kulturdialogs für das Verständnis unter den Völkern auf beiden Seiten des Eisernen Vorhangs. Es erhöhten sich die Möglichkeiten auch der kleinen Völker, ihren Beitrag zu leisten, vor allem mit ihren kulturellen Kontakten. ${ }^{9}$

Der Helsinkiprozess am Beginn der 70er Jahre schuf in Europa eine Atmosphäre aktiver, internationaler Verhandlungen, erzeugte den Geist eines sich verbreitenden Vertrauens zwischen den Ländern mit verschiedenen gesellschaftlich-ökonomischen und politischen Systemen. Im Rahmen der Initiativen des Ostblocks aktivierte auch Bulgarien seine außenpolitischen Initiativen in Europa und insbesondere auf dem Balkan. Deshalb war es nicht zufällig, dass Ljudmila Živkova eine kulturelle Aktion

5 Protokoll „A“ Nr. 13 der Sitzung des Politbüros des ZK der BKP vom 16. 1. 1968, CDA, Fonds 1, op. 35, a. e. 12.

6 Protokoll der Sitzung des Politbüros der ZK der BKP vom 27. 5. 1969, CDA, Fonds 1 B, op. 35 , a. e. 704

7 CDA, op. 26, a. e. 250.

8 CDA, op. 25 , a. e. 255.

9 Ilijana Marčeva, Ljudmila Živkova - Prosvetenijat absoljutizăm [Ljudmila Živkova - Der aufgeklärte Absolutismus], in: Državnicite na Bălgarija sled Vtorata svetovna vojna [Die Staatsmänner Bulgariens nach dem 2. Weltkrieg], Sofija 2003, 1. 
für die Öffnung Bulgariens zur Welt unternahm, zur Popularisierung der bulgarischen kulturellen Errungenschaften, zur Bekanntmachung und Einschätzung kultureller (und nicht klassenmäßig-parteilicher) Positionen der kulturellen Phänomene in der westeuropäischen Zivilisation.

Die ersten Aktionen Ljudmila Živkovas waren Gespräche mit Ministern der Kultur der Länder Europas, Asiens und Afrikas. Sie förderte große Ausstellungen der bulgarischen Kunst in der ganzen Welt, organisierte zahlreiche internationale Symposien, kulturelle und wissenschaftliche Foren in Bulgarien: den Kongress für Bulgaristik, internationale Schriftstellertreffen und andere. In ihrer Zeit wurden neue bulgarische Kulturzentren im Ausland eröffnet. Sie versuchte, schöne Gebäude für die Bedürfnisse der bulgarischen Kultur, auch im Ausland, zu sichern. In ihrer Zeit kaufte die bulgarische Regierung das Haus des weltbekannten Gelehrten Ludwig Wittgenstein in Wien und verwandelte es in ein Kulturzentrum. ${ }^{10}$ Bald nachdem die Ausstellung über thrakische Kunst in der Welt gezeigt wurde, wurde auch die Ausstellung „1000 Jahre bulgarische Ikone“ präsentiert, die als weiterer Beweis des bulgarischen Beitrags zur Weltkultur dienen sollte. ${ }^{11}$

Zur Eröffnung der Ausstellung „Thrakische Kunst“ in Wien in Anwesenheit von Bundespräsident Dr. Rudolf Kirchschläger sagte Ljudmila Živkova: „Das bulgarische Volk ist zu Recht stolz darauf, ein Erbe der thrakischen Zivilisation und Kultur zu sein. Die historische Wissenschaft muss noch den wirklichen Platz und den Einfluss dieser alten und faszinierenden Kultur in der Entwicklung der menschlichen Kultur, insbesondere der europäischen Kultur bestimmen. Unser Volk wird in Kürze den 1300. Jahrestag der Gründung des bulgarischen Staates feiern. Die thrakische Kultur ist einer der ältesten und reichsten Bestandteile der bulgarischen Kultur. Es enthüllt die Jahrtausende alten Wurzeln unserer sozialistischen Kultur. ${ }^{12}$

Das bulgarische Volk kann zurecht ein würdiger Erbe und Nachfolger der schönsten historischen Traditionen, die aus der Antike zu uns gekommen sind, genannt werden. Unter den Bedingungen des sozialistischen Bulgariens wurden diese Traditionen nicht nur bewahrt, sondern auch bereichert und mit einem neuen humaneren Inhalt erfüllt. Die bulgarische nationale Kultur wurde auf eine breitere demokratische Basis gestellt. Eine Nation, die ein integriertes, komplexes, Nationalprogramm für ästhetische Erziehung aufbaut, ist stolz und bewusst, um sich selbst als wirklicher Erbe dieser entfernten Vorfahren zu fühlen, die diese einzigartigen Meisterwerke der menschlichen Kultur und des Fortschritts hinterlassen haben." ${ }^{113}$

Ljudmila Živkova betrachtete die Hochkultur als Mittel zur Durchsetzung eines neuen Bewusstseins. Sie bestand auf dem langfristigen Komplexprogramm für ästhetische Erziehung, auf der Verbreitung einer elitären Kultur und elitärer Formen

${ }_{10}$ Marčeva, Ljudmila Živkova, a. a. O., 6.

11 Marčeva, Ljudmila Živkova, a. a. O., 8.

12 Zhivkova: Her many Worlds, a. a. O., 62.

13 Zhivkova: Her many Worlds, a. a. O., 63. 
der Kunst. Dieses Programm wurde vom 7. Plenum des Komitees für Kunst und Kultur am 6. Dezember 1975 unter der Bezeichnung „Gesamtnationales Programm für ästhetische Erziehung" beschlossen.

Am 30. 5. 1979 beschloss das Sekretariat des ZK der BKP:

1. Das Sekretariat nimmt die von den Genossen Ljudmila Živkova und Petăr Mladenov vorgelegte Information über die Tätigkeit zur Erwerbung von kulturellen und historischen Denkmälern und Werten im Jahr 1978 an;

- Das Sekretariat beschließt die Verwirklichung großer komplexer kulturpropagandistischer und wissenschaftlich-propagandistischer Veranstaltungen im Ausland und insbesondere in Wien, Rom und Toronto. ${ }^{14}$

Die Kinderassemblée „Banner des Friedens“, die nach dem Tod Ljudmilas noch einige Zeit fortgesetzt wurde, war ein weiteres einzigartiges Ereignis im bulgarischen Kulturleben. Sie nahm das Faktum zum Anlass, dass die UNO das Jahr 1979 zum Internationalen Jahr des Kindes erklärte, und Ljudmila beschloss, dieses Forum zu einem ständigen zu machen und Bulgarien zum Initiator und Hausherrn dieser internationalen Veranstaltung. In der Vorbereitung und Durchführung der Kinderassemblée legte Ljudmila ihre ganze Energie hinein. In ihrem Namen wurden Einladungen für die Teilnahme an Intellektuelle ausgeschickt, u. a. an Herbert von Karajan, der 1979 dem Initiativkomitee beitrat. In einer Reihe von Ländern wurden Initiativkomitees eingerichtet. $^{15}$

Nach dem Tod von Ljudmila Živkova verloren die Kulturpolitik, die internationale kulturelle Tätigkeit und die Propaganda den großen Maßstab und ihren Schwung. Obwohl es Parteilinie in der Kultur war, die Ideen von Ljudmila Živkova zu verwirklichen, wurden die meisten stillschweigend aufgegeben. Denn sowohl die innenpolitische und finanzielle Lage des Landes als auch das internationale Klima in den 80er Jahren waren bereits anders und ungünstig für die missionarischen Ansprüche der bulgarischen Kulturpolitik.

Ljudmila Živkova spielte eine große Rolle für die Öffnung der bulgarischen Kultur zur Welt, für die Bereicherung ihrer Kontakte mit verschiedenen Kulturen. Sie tat auch viel für die Popularisierung des bulgarischen Beitrags zur Weltkultur. ${ }^{16}$

Die Kulturpolitik Ljudmila Živkovas kann im Grunde nicht mehr als Variante der sowjetischen Kultur verstanden werden, sondern als eine eigenständige Tendenz, geradezu als Bruch mit der sowjetischen Kultur. ${ }^{17}$ Ihre internationale kulturelle Tätigkeit kann als Politik der „offenen Tür“ zur Welt bezeichnet werden. ${ }^{18}$ Ihr Pro-

${ }^{14}$ Protokoll Nr. 507 des Sekretariats des ZK der BKP vom 30. 5. 1979, CDA, f., op., a. e. 806, 1. 42-45.

15 Schreiben Ljudmila Živkovas an Herbert von Karajan vom 11. 12. 1978, CDA, f. 288, p 2, 1. 48.

16 Marčeva, Ljudmila Živkova, a. a. O., 16.

17 Kiril Avramov, Kulturna politika na Ljudmila Živkova, Sofia 1997 (unveröffentlichtes Manuskript), 2.

18 Avramov, a. a. O., 5. 
gramm stellte sich das ambitionierte Ziel, das schöpferische Prinzip in jedem Menschen zu wecken und soziale Bedingungen für die Entwicklung, Entfaltung und Realisierung der schöpferischen Fähigkeiten im Menschen zu schaffen.

Ljudmila Živkova wollte das nationale Selbstbewusstsein der Bulgaren heben, indem sie bewies, dass auch sie der Welt etwas gegeben hatten. Zu diesem Zweck organisierte sie Ausstellungen wie ,Thrakische Kunst und Kultur in den bulgarischen Gebieten“, die zum ersten Mal 1972 in Sofia gezeigt wurde. Diese Ausstellung wurde international berühmt und wanderte durch 27 Hauptstädte und brachte die Gelehrten dazu, die Kunstgeschichte zu korrigieren. Es folgten die Ausstellungen „1000 Jahre bulgarische Ikone, 9. bis 19. Jahrhundert“, „Mittelalterliches Bulgarien“", u. a.

Die Assemblée „Banner des Friedens“ war das liebste Kind Ljudmila Živkovas. Die erste Assemblée wurde von 15. bis 25. August 1979 in Sofia abgehalten. In diesen Tagen trafen sich 1100 bulgarische und 1321 ausländische Kinder aus 76 Ländern, die rund 6000 Werke vorstellten, etwa hundert musikalische Werke aufführten und 160 literarische Werke präsentierten. Die Assemblée war eine Idee Svetoslav Roerichs, des Sohnes Nikolaj Roerichs. Die Assemblée hob die Rolle Bulgariens als Brücke zwischen Europa und Asien hervor und betonte die Eigenständigkeit der Bulgaren.

Im Jahr 1981 wurde auf Initiative Ljudmila Živkovas die Feier zum 1300jährigen Bestandsjubiläum des bulgarischen Staates abgehalten. Die Sowjets wollten die Feier verhindern, weil sie sie als nationalistische Veranstaltung betrachteten, aber Ljudmila Živkova sprach mit Leonid Brežnev persönlich und erhielt die Erlaubnis.

Ljudmila Živkovka betonte: „Wir brauchen diese Öffnung. In der Welt entwickeln sich gesellschaftliche und wissenschaftlich-technische Prozesse, hinter denen wir zurückbleiben. Deshalb werden wir uns enger mit ihnen verbinden. “19

Die nonkonformistischen oder direkt häretischen Ideen Živkovas wurden besonders deutlich sichtbar, als sie Mitglied der Regierung und später des Politbüros geworden war. Ljudmila Živkova hatte sich die strategische Aufgabe gestellt, alle philosophischen, ethischen und ästhetischen Postulate des Marxismus-Leninismus, die in jener Zeit dem gesellschaftlichen Leben als unabänderliches Diktat auferlegt waren, schrittweise an die letzte Stelle zu setzen und danach völlig abzuschaffen. ${ }^{20}$

Das Büro des Komitees für Kultur entwickelte einen Fünfjahresplan für die Kultur:

„Der erste Fünfjahresplan für unsere informativ-propagandistische, wissenschaftliche, kulturelle und bildungsmäßige Tätigkeit im Ausland stellt einen Teil des 7. Fünfjahresplans (1976-1980) dar. Dieser Plan ergibt sich aus den Beschlüssen und

${ }^{19}$ Emil Aleksandrov, Plažna sbirka planira nov zlaten vek [Die Gruppe am Strand plant ein Goldenes Zeitalter], 24 časa, 26. 9. 1997, 9.

20 Bogomil Rajnov, Ljudmila: mečti i dela [Ljudmila: Träume und Werke], Sofia 2003, 3739. 
Aufgaben, die vom 10. Parteitag der BKP, vom Juliplenum 1973 und dem Februarplenum 1974 sowie von den Plenarsitzungen des ZK der BKP vom 25. 5. 1969 und vom 26. 3. 1974, den Empfehlungen der Moskauer und Prager Beratungen der Sekretäre, der Zentralkomitees, der kommunistischen Parteien der sozialistischen Länder für ideologische Arbeit und außenpolitische Propaganda (1973) und der Märzberatung für Fragen der außenpolitischen Propaganda bei uns (1974) gestellt wurden. ${ }^{\text {2 }} 1$

\section{Das Bulgarische Forschungsinstitut in Österreich}

Die besondere Rolle Österreichs für Bulgarien wurde durch die Gründung des bulgarischen Kulturzentrums im Haus Wittgenstein in Wien im Dezember 1976 (eröffnet am 4. 1. 1977 durch ein Konzert in Anwesenheit von Frau Minister Hertha Firnberg) besonders charakterisiert. Das Haus Wittgenstein, im Jahr 1928 von dem Philosophen Ludwig Wittgenstein für seine Schwester Margarete im Stil der „Neuen Sachlichkeit" erbaut, auf Initiative von Botschafter Vladimir Ganovski vom bulgarischen Staat angekauft und nach Plänen von Carl Auböck restauriert, entwickelte sich mit der Kulturabteilung der Bulgarischen Botschaft und dem Bulgarischen Forschungsinstitut in Österreich in den siebziger und achtziger Jahren zu einem Mittelpunkt des Wiener Kulturlebens. ${ }^{22}$

Das Bulgarische Forschungsinstitut in Österreich beruhte auf den Beschlüssen des Sekretariats des ZK der BKP vom 19. 4. 1975:

„Das Ministerium für Finanzen soll in Übereinstimmung mit der Abteilung „Kader, Parteiorganisationen und Auslandsreisen“ des ZK der BKP das Auslandspersonal des Ministeriums für Auswärtige Angelegenheiten um zehn Personen zur Errichtung von ständigen Forschungsgruppen für Bulgaristik in den nichtsozialistischen Ländern erhöhen.

Das Ministerium für Auswärtige Angelegenheiten, das Komitee für Kultur und die Bulgarische Akademie der Wissenschaften sollen bis 15. September 1977 eine Forschungsgruppe für Bulgaristik in Wien im Rahmen des wissenschaftlichen und kulturellen Komplexes Haus Wittgenstein errichten. ${ }^{\text {223 }}$

Das Sekretariat des ZK der BKP beschloss ferner ein Provisorisches Statut über die Aufgaben, die Struktur und die Tätigkeit der Forschungsgruppe für Bulgaristik in Österreich - Wien:

„1. Die Forschungsgruppe für Bulgaristik in Österreich - Wien, gegründet am 11. 9. 1977, gemäß Punkt 5 des Beschlusses des Sekretariats des ZK der BKP Nr. 872, ist eine Einrichtung der Leitung bei dem Ministerium für Auswärtige Angele-

${ }^{21}$ CDA, F 405, op. 10, a. e. 78, 50.

22 O. Kapfinger: Haus Wittgenstein - eine Dokumentation. Kulturabteilung der Botschaft der Republik Bulgarien. Wien 1991.

23 Beschluss Nr. 872 des Sekretariats des ZK der BKP vom 11.9.1977, CDA, F, op., a. e. $1325,1.2-3$. 
genheiten, dem Komitee für Kultur und der Bulgarischen Akademie der Wissenschaften.

2. Die Hauptaufgabe der Gruppe für Bulgaristik besteht darin, Forschungen über die gegenseitigen historischen, kulturellen und sprachlichen Einflüsse zwischen unserem und anderen Völkern in Europa durchzuführen. Das Ziel der Forschungen besteht darin, die allgemein menschlichen (universellen) Werte in der Entwicklung der bulgarischen Geschichte und Kultur zu bekräftigen, die Errungenschaften des bulgarischen historischen Denkens zu propagieren, den Platz des bulgarischen Volkes, seiner Sprache und Kultur in der Weltgeschichte aufzuzeigen und das wissenschaftliche und kulturelle Ansehen der VRB hauptsächlich in der kapitalistischen Welt zu heben.

3. Die Gruppe für Bulgaristik hat folgende grundlegende Aufgaben:

- Funktionen als ausländischer Koordinator der Forschungen über Bulgaristik in den europäischen kapitalistischen Ländern zu verwirklichen;

- eine Verlagstätigkeit durch eigene Ausgaben (eine monographische und operative Reihe) und durch Ausgaben in anderen Ländern zu organisieren und zu verwirklichen;

- der Festigung von bulgaristischen Spezialisten in den europäischen kapitalistischen Ländern zu helfen;

- internationale wissenschaftliche Tagungen über aktuelle Probleme der bulgarischen Geschichte und der zeitgenössischen bulgarischen Kultur zu organisieren;

- die komplexe wissenschaftliche und propagandistische Verteidigung der grundlegenden Thesen der Geschichte, Kulturgeschichte und des gegenwärtigen sozialistischen Aufbaus in den kapitalistischen europäischen Ländern zu organisieren;

- wissenschaftliche Expeditionen in die Regionen mit bulgarischer Bevölkerung oder Spuren bulgarischer Kultur und Sprache zu organisieren;

- außenpolitische, wissenschaftspolitische und kulturpolitische Information für die drei Behörden durch entsprechende Informations- und Dokumentationsmaterialien auszuwählen, zu bearbeiten und zur Verfügung zu stellen.“ ${ }^{24}$

Das Denkmal für die Heiligen Kyrill und Method im Garten des Hauses Wittgenstein, geschaffen vom Bildhauer Prof. Dimităr Bojkov, wurde im September 1978 von Ljudmila Živkova in Anwesenheit ihres Vaters, Staats- und Parteichef Todor Živkov, und des Botschafters Stojan Georgiev enthüllt.

In dieser Periode wurde eine Reihe von Direktkontakten zwischen Forschungs-, Bildungs- und Kulturorganisationen auf vertragsrechtliche Grundlage gestellt. Hierzu gehörten die Abkommen zwischen der Österreichischen und der Bulgarischen Akademie der Wissenschaften (1977), zwischen dem Österreichischen Rundfunk und dem bulgarischen Fernsehen (1979), zwischen dem Künstlerhaus Wien und dem

24 Provisorisches Statut über Aufgaben, Struktur und Tätigkeit der Forschungsgruppe für Bulgaristik in Österreich - Wien, 14. 9. 1977, CDA, f., op., a. e., 1. 1-4. 
Verband bulgarischer Künstler (1981) sowie zwischen der Österreichischen Vereinigung für moderne Musik und dem Verband der bulgarischen Komponisten (1982). ${ }^{25}$

Der Leiter der Kultursektion des bulgarischen Außenministeriums, Christo Santov, erklärte deshalb dem Schreiber dieser Zeilen:

„Die kulturellen Beziehungen zu Österreich sind die am besten entwickelten, die Bulgarien mit der westlichen Welt unterhält. “26

Das Bulgarische Forschungsinstitut in Österreich (BFIÖ) wurde durch einen Beschluss des Sekretariats des ZK der BKP vom 11. 11. 1977 gegründet, aber die Gründung des österreichischen Vereins „Freunde des Hauses Wittgenstein“, des Trägervereins des Instituts, erfolgte erst am 20. 1. 1978 auf einer Generalversammlung, unter dem Vorsitz von Prof. Carl Auböck.

Von 1978 bis 1989 veranstaltete das BFIÖ allein oder in Kooperation mit österreichischen Instituten 31 internationale wissenschaftliche Tagungen in Wien, Graz, Salzburg, Sofia, Chaskovo, Dobrič und 44 Vortragsabende im Rahmen seines Lectorium Bulgaricum, zahlreiche Dokumentarausstellungen, Buchpräsentationen usw.

Auf Initiative von Ljudmila Živkova, die Frau Minister Hertha Firnberg für die Idee gewinnen konnte, wurde im Jahr 1981 das in Salzburg bereits bestehende Institut für Slawistik mit der besonderen Pflege der Bulgaristik beauftragt. Das Institut, dessen damaliger Direktor, Univ. Prof. Dr. Otto Kronsteiner seit 1982 die Zeitschrift „Die Slawischen Sprachen“ herausgab und einmal jährlich das „Salzburger Slawistengespräch" veranstaltete, arbeitete an der kritischen Edition altbulgarischer Texte, insbesondere der Bibelübersetzung des Hl. Method. ${ }^{27}$

Eine wesentliche Rolle in den Wissenschaftsbeziehungen zwischen Österreich und Bulgarien spielte das Österreichische Ost- und Südosteuropa-Institut (OSI), das besonders in den 80er Jahren eine intensive Zusammenarbeit mit dem BFIÖ und Bulgarien entwickelte. Im November 1978 begann mit einer gemeinsamen österreichisch-bulgarischen Konferenz über „Archäologische Probleme im heutigen Siedlungsraum“, gefolgt von einer Arbeitstagung des Centre International d'Information sur les Sources de l'Histoire Balkanique et Méditerranéenne unter dem Vorsitz von Prof. Dr. Nikolaj Todorov eine neue, intensive Phase der Zusammenarbeit insbesondere mit dem Bulgarischen Forschungsinstitut. ${ }^{28}$

25 V. Antov: Kulturbeziehungen Bulgarien-Österreich, Sofia Press Bulletin, Okt. 1989.

${ }^{26}$ C. Santov, Leiter der Kultursektion des bulgarischen Außenministeriums, am 27. 4. 1987 in Sofia.

27 Die Slawischen Sprachen, hrsg. von O. Kronsteiner, Bd. 34, 1993.

28 Tätigkeitsberichte des Österreichischen Ost- und Südosteuropa-Instituts in den Österreichischen Ostheften Nr. 1 (1963), Nr. 1 (1979), Nr. 1 (1983), Nr. 1 (1985), Nr. 1 (1989) und Nr. 1 (1992). 
DAS JUBILÄUM „1300 JAHRE BulgarieN“

Die Veranstaltungen des Hauses Wittgenstein und des Bulgarischen Forschungsinstituts wurden von ihrer Gründung and im Hinblick auf das Jubiläum „1300 Jahre Bulgarien" organisiert. Mit dem Künstlerhaus in Wien gab es seit den 70er Jahren sehr enge Beziehungen. Es wurde eine Ausstellung von Vladimir Dimitrov-Majstora veranstaltet und ein Vertrag zwischen dem österreichischen und dem bulgarischen Künstlerverband abgeschlossen. Der Präsident des Künstlerhauses, Hans Meyer, war mit Dečko Uzunov befreundet, und es gab einige bulgarische Ausstellungen im Künstlerhaus. Die österreichischen Künstler Adolf Frohner und Peter Kudera besuchten Bulgarien. Der bulgarische Kulturattaché in Wien, Christo Drumev, war in dieser Sache sehr aktiv. Der Industrielle Peter Ludwig spielte in diesem Zusammenhang eine große Rolle, weil er zahlreiche Werke bulgarischer Künstler ankaufte. Georgi Jordanov unterstützte diese Kontakte und nahm an der Eröffnung bulgarischer Ausstellungen in Wien teil. ${ }^{29}$

Ivan Charalambiev, der Vorsitzender der nationalen Koordinationskommission „1300 Jahre Bulgarien“, sagte am 26. 8. 1980 in einer Sitzung der Kommission: „Unser Interesse an der heutigen Tagung ist gerechtfertigt im Hinblick auf den besonderen Platz, den unsere diplomatische Mission und dementsprechend das in Österreich existierende Nationalkomitee in Zusammenhang mit der Tätigkeit für die 1300-Jahr-Feier Bulgariens einnimmt. Die Rede ist von einem Land, wo die Aktivitäten ein besonders hohes Niveau haben. Wenn wir die Ergebnisse unserer bisherigen internationalen Tätigkeit hervorheben, beginnen wir gewöhnlich mit der Republik Österreich.“30

„Es ist kein Zufall, dass vor zwei Jahren (1978), als der Rechenschaftsbericht in der Volksversammlung gehalten wurde, die Republik Österreich in einem eigenen Absatz erwähnt wurde, in dem es hieß, dass die Beziehungen zwischen Österreich und Bulgarien ein Beispiel von wechselseitigen Beziehungen zwischen zwei Ländern mit unterschiedlicher gesellschaftlich-ökonomischer Ordnung sein sollen. Heute sind sie ein Beispiel.“31

Die Jubiläumsfeier zum 1300jährigen Bestehen des bulgarischen Staates, die in einem Zeitraum von vier Jahren (1978-1981) begangen wurde, war von großer politischen Bedeutung, weil sie so konzipiert war, dass die Unabhängigkeit Bulgariens stark zum Ausdruck kam. Das Projekt führte zu einem Konflikt mit den Sowjets, die von Todor Živkov verlangten, mit dem ganzen Prozess aufzuhören.

Das Festkonzert in der Staatsoper in Wien am 14. Juni 1981 in Anwesenheit des Bundespräsidenten Kirchschläger, der Ministerin für Wissenschaft und Forschung

${ }^{29}$ Gespräch mit Svetlin Rusev in Wien am 9. 2. 2005.

30 Erörterung der Vorbereitung für die 1300-Jahr-Feier des bulgarischen Staates in der Republik Österreich, nationale Koordinationskommission „1300 Jahre Bulgarien“, 26. 8. 1980, CDA, F 990, op. 1, a. e. 454, 3-4.

31 CDA, F 990, op. 1, a. e. 454, 6. 
Hertha Firnberg, des Innenministers Karl Blecha, der auch Präsident der Österreichisch-bulgarischen Gesellschaft war, und der halben österreichischen Regierung sowie 1400 Festgästen war der Höhepunkt der österreichisch-bulgarischen Kulturbeziehungen. Die Festansprache wurde von Ljudmila Živkova gehalten, die in Wien ihre letzte öffentliche Rede im Ausland hielt.

In der Periode der Jubiläumstätigkeit registrierte die Botschaft keine Versuche örtlicher Organisationen in Richtung Widerstand gegen das bulgarische Jubiläum und Verminderung des Effekts der Jubiläumsveranstaltungen. Im Jahre 1981 gab es keinerlei politische Demonstrationen gegen Bulgarien, obwohl es zur selben Zeit Kundgebungen gegen die Unterdrückung der Opposition in Polen, der DDR, der Tschechoslowakei und Rumänien gab. ${ }^{32}$

Unter den Veranstaltungen in der Vorbereitung des Jubiläums sind zu erwähnen: das Gastspiel des bulgarischen Nationaltheaters „Ivan Vazov“ im Burgtheater mit zwei Aufführungen am 15. und 16. 10. 1979 und zehn Aufführungen der Sofioter Oper (Nationales Akademisches Theater für Oper und Ballett) in der Wiener Staatsoper im November 1979 sowie der dritte internationale Kongress für Thrakologie vom 2. bis 6. 6. 1980 in der österreichischen Akademie der Wissenschaften unter dem Vorsitz von Prof. Herbert Hunger. Die bulgarische Delegation wurde vom Minister für Volksbildung Prof. Alexander Fol, der auch Direktor des Instituts für Thrakologie in Sofia war, angeführt. ${ }^{33}$

Die Zeitschrift „Österreichische Osthefte“ veröffentlichte 1981 eine Sondernummer mit Beiträgen über Geschichte, Kultur und Literatur anlässlich des Jubiläums des bulgarischen Staates. Das war einmalig, denn ein Sonderband über andere Ostblockländer aus Anlass eines Jubiläums wäre damals (mit Ausnahme Ungarns) nicht möglich gewesen. ${ }^{34}$

Durch den Tod Ljudmila Živkovas am 21. Juli 1981 kam es zu einer gewissen Einschränkung der Aktivitäten, weil Bulgarien für die Kultur nicht mehr dasselbe Budget zur Verfügung stellte, und auch weil Bundeskanzler Kreisky 1983 zurücktrat.

Im Mai 1984 kam der Kandidat des Politbüros und Vorsitzende des Komitees für Kultur Georgi Jordanov im Zusammenhang mit einer Sitzung der „Internationalen Stiftung Ljudmila Živkova“ nach Wien. Auf der Grundlage des verwirklichten kulturellen Austausches im Jahr 1984 kann man den Schluss ziehen, dass trotz einiger Veränderungen unter dem Einfluss von inneren und äußeren Faktoren der Hauptinhalt und die Richtung der Beziehungen zwischen Österreich und Bulgarien ihren Charakter beibehielten. ${ }^{35}$

${ }^{32}$ CDA, F 990, op. 1, a. e. $459,15$.

33 CDA, F 990, op. 1, a. e. 459, 54.

34 CDA, F 990, op. 1, a. e. 459, 74.

35 Bericht über die Tätigkeit der einheitlichen diplomatischen Vertretung der VRB in Wien im Jahr 1984, 16. 1. 1985, CPA, P-317, 1. 7. 
Besondere Aufmerksamkeit verdiente die aktive Tätigkeit der Österreichischbulgarischen Gesellschaft. Die Beziehungen wurden auf höchster Ebene unterhalten. Aus Anlass des 30. Jahrestages ihrer Gründung weilte 1986 eine bulgarische Delegation in Wien, geleitet vom stellvertretenden Vorsitzenden des Staatsrats und Vorsitzenden der bulgarisch-österreichischen Gesellschaft Georgi Džagarov, der auch von Bundespräsident Waldheim empfangen wurde. ${ }^{36}$

A b stra c t: The Cultural Propaganda of the People's Republic of Bulgaria in Austria. This article deals with the Austrian Eastern policy of the Kreisky era, Bulgarian cultural foreign policy toward Western countries, the cultural policy of Lyudmila Zhivkova, the cultural relations of Bulgaria with Austria, the Bulgarian cultural centre "House Wittgenstein" in Vienna and the 1300th anniversary of the Bulgarian state in 1981. kova

K e y w ords: Bulgarian cultural policy, Bulgarian-Austrian relations, Lyudmila Zhiv-

Peter Bachmaier

Bulgarisches Forschungsinstitut in Österreich

Parkgasse 18

1030 Wien, Österreich

p.bachmaier@aon.at

${ }^{36}$ Bericht über das Jahr 1986, 9. 2. 1987, CPA, P-561, 1. 16-24. 